\title{
The complete targeted profile of the organic acid intermediates of the citric acid cycle using a single stable isotope dilution analysis, sodium borodeuteride reduction and selected ion monitoring GC/MS
}

\author{
Orval Mamer $\cdot$ Simon-Pierre Gravel $\cdot$ \\ Luc Choinière $\cdot$ Valérie Chénard · Julie St-Pierre \\ Daina Avizonis
}

Received: 21 November 2012/ Accepted: 15 March 2013/Published online: 17 April 2013

(C) Springer Science+Business Media New York 2013

\begin{abstract}
The quantitative profiling of the organic acid intermediates of the citric acid cycle (CAC) presents a challenge due to the lack of commercially available internal standards for all of the organic acid intermediates. We developed an analytical method that enables the quantitation of all the organic acids in the CAC in a single stable isotope dilution GC/MS analysis with deuterium-labeled analogs used as internal standards. The unstable $\alpha$-keto acids are rapidly reduced with sodium borodeuteride to the corresponding
\end{abstract}

Orval Mamer and Simon-Pierre Gravel contributed equally to this study.

Electronic supplementary material The online version of this article (doi:10.1007/s11306-013-0521-1) contains supplementary material, which is available to authorized users.

O. Mamer $\cdot$ L. Choinière

Metabolomics Core Facility, Rosalind and Morris Goodman Cancer Research Centre, McGill University, Montreal,

QC, Canada

S.-P. Gravel · V. Chénard

Department of Biochemistry and Rosalind and Morris Goodman Cancer Research Centre, McGill University, Montreal,

QC, Canada

J. St-Pierre $(\bowtie)$

Department of Biochemistry and Rosalind and Morris Goodman Cancer Research Centre, McGill University, 3655 Promenade Sir William Osler, Office: Room 713A, Montreal,

QC H3A 1A3, Canada

e-mail: julie.st-pierre@mcgill.ca

D. Avizonis $(\bowtie)$

Metabolomics Core Facility, Rosalind and Morris Goodman Cancer Research Centre, McGill University, 1160 Pine Avenue West, Room 418, Montreal, QC H3A 1A3, Canada

e-mail: daina.avizonis@mcgill.ca stable $\alpha$-deutero- $\alpha$-hydroxy acids and these, along with their unlabeled analogs and other CAC organic acid intermediates, are converted to their tert-butyldimethylsilyl derivatives. Selected ion monitoring is employed with electron ionization. We validated this method by treating an untransformed mouse mammary epithelial cell line with well-known mitochondrial toxins affecting the electron transport chain and ATP synthase, which resulted in profound perturbations of the concentration of CAC intermediates.

Keywords GC/MS $\cdot$ Citric acid cycle $\cdot$ Metabolite profiling $\cdot$ Mitochondrial toxins

\section{Introduction}

Mitochondrial functions have been traditionally determined using in vitro assays, notably quantification of oxygen consumption, enzyme activities and membrane potential measurements. The quantitative measurement of the organic acids associated with the citric acid cycle (CAC) is of great interest not only due to its intimate coupling to the electron transport chain and to oxidative phosphorylation, but also more recently, due to its altered activity in cancer cells (Mullen et al. 2012) and a renewed interest in the chemistry of the Warburg effect (Vander Heiden et al. 2009). The development of novel technologies that permit the precise quantitative evaluation of mitochondrial metabolite abundances represents an important new avenue of research for the monitoring of mitochondrial functions in physiological and pathological conditions (Fiehn 2001; Steuer et al. 2003).

The measurement of the CAC intermediates has been a scientific goal for chemists for a significant period of time. Early liquid or gas chromatography and mass spectrometry 
studies resulted in difficult quantification of the CAC intermediates and poorly resolved peaks. Notably, several techniques resulted in multiple peaks for the unstable keto acids and did not resolve citric and isocitric acids (Busch et al. 1952; Dalgliesh et al. 1966; Harmon and Doelle 1969; Turkelson and Richards 1978; Zaura and Metcoff 1969). One complicating factor in analyzing the $\alpha$-keto acids (oxaloacetic, $\alpha$-ketoglutaric, and pyruvic acids) is that they may not be stable under sample extraction conditions and can spontaneously decarboxylate (Tsai 1967). A solution to quantitating unstable $\alpha$-keto acids is to convert them to their much more stable corresponding $\alpha$-hydroxy acids with hydride reducing agents. For example, sodium borodeuteride reduction of the branched chain $\alpha$-keto acid metabolites of valine, leucine and isoleucine has been reported to simplify their determination along with their corresponding $\alpha$-hydroxy acid metabolites in human serum using a stable isotope dilution assay (Mamer et al. 1986). Similarly, urinary pyruvic and acetoacetic acids can be quantitated by reduction with sodium borodeuteride to singly labeled lactic and $\beta$-hydroxybutyric acids, which can be measured along with their unlabeled endogenous analogues (Mamer 1988).

In recent years, several techniques have emerged that could measure CAC intermediates with greater precision, albeit still with limited coverage. In particular these include LC-MS/MS techniques developed by Luo et al. 2007 (Luo et al. 2007) and Koubaa et al. 2013 (Koubaa et al. 2013), GC/MS methodologies (Dunn and Winder 2011; Kombu et al. 2011), HPLC-fluorescence methodology (Kubota et al. 2005), capillary electrophoresis coupled to a mass spectrometer (CE/MS) (Soga et al. 2003; Wakayama et al. 2010), as well as ${ }^{1} \mathrm{H}$ NMR (Xu et al. 2011). ${ }^{1} \mathrm{H}$ NMR permits a greater coverage of the CAC intermediates as well as other metabolites (Xu et al. 2011) and does not require isotopically labeled internal standards. However, it is limited by the low micromolar sensitivity and potential spectral overlap. Furthermore, CAC metabolites such as $\alpha$-ketoglutaric, citric, isocitric and cis-aconitic acids are frequently difficult to detect because of their low abundance (Chatham et al. 2003).

Currently there are varied techniques for measuring CAC intermediates, each having coverage and/or sensitivity issues. In this paper, we report the development of a simple, comprehensive, targeted and quantitative technique that quantifies all organic acid intermediates of the CAC, addresses the issues of producing one quantifiable peak per metabolite, provides good chromatographic resolution, and addresses the stability of the $\alpha$-keto acids. Our approach is based on stable isotope dilution selected ion monitoring (SIM) GC/MS. The $\alpha$-keto acids, pyruvic, oxaloacetic, $\alpha$-ketoglutaric and oxalosuccinic acids are simply extracted under ice cold conditions then quickly stabilized by reduction with sodium borodeuteride to form singly deuterium-labeled hydroxy acids as shown in the following reaction scheme:

Reaction of $\alpha$-Keto Acids with Sodium Borodeuteride

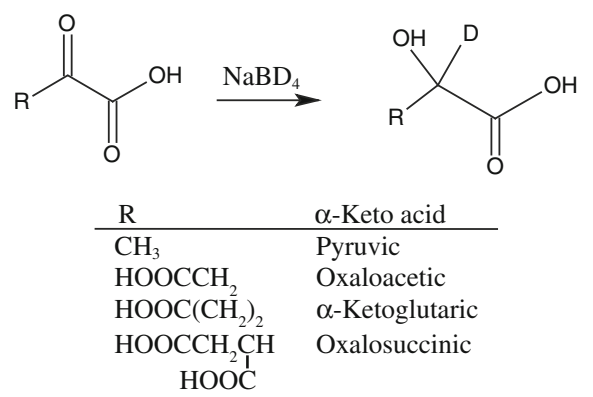

This reduction converts the relatively unstable $\alpha$-keto acids to the much more stable corresponding $\alpha$-hydroxy- $\alpha$ deutero acids for GC/MS analysis. Furthermore, it reduces the number of isotopically labeled analogs necessary for quantitative stable isotope dilution experiments, all of which are commercially available. The resulting acids are converted to their tert-butyldimethylsilyl (TBDMS) derivatives to take advantage of the intense $\left[\mathrm{M}^{+\cdot}-\mathrm{C}_{4} \mathrm{H}_{9}\right]^{+}$ fragment ions observed in the spectra of these intermediates and the improved resolution between citric and isocitric acids. We illustrate the use of this method in the quantitation of the complete CAC organic acid intermediates in an untransformed mouse mammary epithelial cell line (NMuMG) under basal conditions or treated with various mitochondrial electron transport chain inhibitors. The ability to precisely associate patterns of CAC perturbations to a specific inhibition in the electron transport chain will facilitate the evaluation of mitochondrial functions in physiology and disease.

\section{Experimental methods}

\subsection{Tissue culture and treatments}

Antimycin A (A8674), oligomycin (O4876) and rotenone (R8875) were purchased from Sigma-Aldrich Canada Ltd., Oakville, Ontario, Canada. NMuMG cells were obtained from the American Type Culture Collection (ATCC). Cells were grown in an incubator maintained at constant temperature $\left(37{ }^{\circ} \mathrm{C}\right)$ and humidity, with $5 \% \mathrm{CO}_{2}$ supply. Cells were grown in Dulbecco's Modified Eagle Medium (DMEM) (Wisent Inc, St. Bruno, Quebec, Canada) supplemented with $10 \%$ fetal bovine serum (Wisent Inc.), $10 \mu \mathrm{g} / \mathrm{mL}$ insulin, $20 \mathrm{mM}$ HEPES pH 7.2 and penicillin/ streptomycin. NMuMG cells were grown to $80 \%$ confluence $(\sim 3,000,000$ cells) then trypsinized and counted with a TC10 automated cell counter (Bio-Rad, Hercules, CA, USA). For each treatment, 3-5 dishes were used for CAC 
analysis by GC/MS, while 2-3 dishes were used for cell counting. Three independent cell passages consisting of three to five replicates were grown in culture. After a period of $18 \mathrm{~h}$ following seeding, the medium was removed and replaced by complete medium containing either DMSO $(0.4 \%$, control), antimycin A $(10 \mu \mathrm{M})$, rotenone $(10 \mu \mathrm{M})$ or oligomycin $\left(2.5 \mu \mathrm{g} / 10^{6}\right.$ cells $)$ for $2 \mathrm{~h}$.

\subsection{Cell extract sample preparation}

The growth medium from each tissue culture plate was aspirated off and discarded. The plates were carefully washed three times with $2 \mathrm{~mL}$ of ice-cold isotonic saline solution $(9 \mathrm{~g} / \mathrm{L})$ and maintained on ice throughout the procedure. Thoroughly washing cells with normal saline significantly reduces the amount of phosphate in the samples. This improves reproducibility from sample to sample and minimizes the free phosphate damage to the GC column. A $500 \mu \mathrm{L}$ volume of $80 \%$ methanol in deionized distilled water cooled to $-80{ }^{\circ} \mathrm{C}$, was rapidly pipetted onto each plate and the cells were scraped loose and transferred to pre-chilled tubes (this step was repeated twice). Cell suspensions were quickly vortexed and then subjected to sonication on ice using the BioRuptor (UCD-200 TM, Diagenode) for $10 \mathrm{~min}$ ( $30 \mathrm{~s} \mathrm{ON}, 30 \mathrm{~s} \mathrm{OFF})$ at the "high" setting. Metabolite suspensions were cleared by centrifugation at $21,000 \mathrm{~g}$ at $4{ }^{\circ} \mathrm{C}$ for $10 \mathrm{~min}$. Each supernatant was transferred into a fresh pre-chilled tube. A $1 \mu \mathrm{L}$ volume of $750 \mathrm{ng} / \mu \mathrm{L} \mathrm{D}_{27}$-myristic acid dissolved in pyridine was added to each sample as a secondary internal standard.

The $\mathrm{pH}$ of the supernatants was adjusted to $\sim 11$ (on ice and confirmed using pH paper) by the addition of $5 \mu \mathrm{L}$ of $2 \mathrm{~N} \mathrm{NaOH}$. A $15 \mu \mathrm{L}$ volume of freshly prepared $10 \mathrm{mg} /$ $\mathrm{mL} \mathrm{NaBD}_{4}$ dissolved in $50 \mathrm{mM} \mathrm{NaOH}$ was added to each sample followed by $20 \mathrm{~s}$ of vortexing. The samples were then moved to and incubated at room temperature for $1 \mathrm{~h}$. After incubation, samples were acidified to $\mathrm{pH} 4$ by the addition of $15 \mu \mathrm{L}$ of $3.5 \mathrm{M} \mathrm{HCl}$. Care was taken to add acid slowly due to deuterium evolution. Each sample was spiked with $1 \mu \mathrm{L}$ of the stable isotope internal standard mixture (Table 1) post-acidification to avoid base catalyzed back exchange of deuterium with hydrogen. Samples were frozen and dried in a vacuum centrifuge (Labconco, Kansas City, MO, USA) at $-4{ }^{\circ} \mathrm{C}$. Methanol $(50 \mu \mathrm{L})$ was added to each sample and evaporated to dryness to remove boric acid as trimethyl borate. Ensuring that all samples, solvents and reagents are completely anhydrous greatly improves reproducibility.

Samples were re-suspended with sonication and vortex mixing in $30 \mu \mathrm{L}$ of pyridine and centrifuged $(>10,000 \mathrm{~g})$ at room temperature for $10 \mathrm{~min}$. The supernatants were transferred to GC/MS autoinjector vials containing $70 \mu \mathrm{L}$ $\mathrm{N}$-tert-butyldimethylsilyl- $\mathrm{N}$-methyltrifluoroacetamide
Table 1 Deuterium-labeled internal standard composition

\begin{tabular}{|c|c|c|}
\hline Labeled acid & $\begin{array}{l}\text { Molecular } \\
\text { weight }\end{array}$ & $\begin{array}{l}\text { Concentration } \\
(\mathrm{nmol} / \mu \mathrm{L})\end{array}$ \\
\hline $3,3,3-\mathrm{D}_{3}$-lactic & 115.1 & 3.44 \\
\hline 2,3- $\mathrm{D}_{2}$-fumaric & 118.1 & 1.03 \\
\hline 2,2,3,3- $\mathrm{D}_{4}$-succinic & 124.1 & 0.89 \\
\hline $2,3,3-\mathrm{D}_{3}$-malic & 137.1 & 0.28 \\
\hline $3,3,4,4-\mathrm{D}_{4}-\alpha$-ketoglutaric & 152.1 & 0.20 \\
\hline 2,2,4,4-D $\mathrm{D}_{4}$-citric & 196.2 & 0.32 \\
\hline Tricarballyic & 176.1 & 1.65 \\
\hline
\end{tabular}

(MTBSTFA) and incubated at $70{ }^{\circ} \mathrm{C}$ for $1 \mathrm{~h}$. A volume of $1 \mu \mathrm{L}$ was injected for $\mathrm{GC} / \mathrm{MS}$ analysis.

\subsection{Authentic standards preparation}

Authentic unlabeled acids, stable isotope labeled standards, anhydrous pyridine and MTBSTFA were purchased from Sigma-Aldrich Canada Ltd. (Oakville, Ontario, Canada), while $\mathrm{NaBD}_{4}$ and sodium 3,3,3-D - -lactate were purchased from CDN Isotopes (Pointe-Claire, Quebec, Canada). The TBDMS derivatives of each organic acid were made and analyzed individually to acquire authentic mass spectra and to define retention times and SIM conditions.

To determine the isotopic purity of $\mathrm{NaBD}_{4}$, a solution of pyruvic, $\alpha$-ketoglutaric and oxaloacetic acids $(\sim 1 \mathrm{ng} / \mu \mathrm{L}$ $20 \mathrm{mM} \mathrm{NaOH}$ ) was quantitatively reduced in $10 \mathrm{~mL}$ of $20 \mathrm{mM} \mathrm{NaOH}$, to which $5 \mathrm{mg}$ of $\mathrm{NaBD}_{4}(99.8$ at.\% D) was added. The resulting solution was stirred until it was homogeneous and held at room temperature for $1 \mathrm{~h}$. The $\mathrm{pH}$ of the solution was then lowered to 4 with $3.5 \mathrm{~N} \mathrm{HCl}$ added drop-wise (caution, deuterium evolution). This quantitatively reduced pyruvic, $\alpha$-ketoglutaric and oxaloacetic acids to $\alpha$ - $\mathrm{D}_{1}$-lactic, $\alpha-\mathrm{D}_{1}-\alpha$-hydroxyglutaric and $\alpha-\mathrm{D}_{1}$-malic acids, respectively. Volumes of 1,3 and $5 \mu \mathrm{L}$ of this mixture were dried under a stream of nitrogen and derivatized as described in Sect. 2.2.

\subsection{Quantitating internal standard solution preparation}

A stock solution of tricarballylic acid and deuteriumlabeled CAC acids was prepared from authentic samples (Table 1). Carefully weighed quantities were dissolved in $80 \mathrm{~mL}$ of $0.05 \mathrm{~N} \mathrm{NaOH}$, to which was added $40 \mathrm{mg}$ of $\mathrm{NaBD}_{4}$. This was held at $50{ }^{\circ} \mathrm{C}$ for $10 \mathrm{~min}$, acidified with $5 \mathrm{~N} \mathrm{HCl}$ to $\mathrm{pH} 4$ (caution: deuterium evolution), diluted to $100 \mathrm{~mL}$ with distilled water and stored at $-30{ }^{\circ} \mathrm{C}$. This quantitatively reduced $3,3,4,4-\mathrm{D}_{4}$ - $\alpha$-ketoglutaric acid to $2,3,3,4,4-D_{5}-\alpha$-hydroxyglutaric acid. Since currently there are no commercially available isotopologues for cis- 
aconitic acid, tricarballylic acid was used as the quantitating standard. It is structurally similar to and elutes very close to cis-aconitic acid. Likewise, labeled and unlabeled oxalosuccinic acids were unavailable commercially, but could be quantitated as $\mathrm{D}_{1}$-isocitric acid against $\mathrm{D}_{4}$-citric acid.

\subsection{Preparation of samples for limits of quantitation calibration}

Four stock solutions were prepared containing $1 \mathrm{mM}$ concentrations of succinic, fumaric, malic, $\alpha$-hydroxyglutaric, cis-aconitic, citric and isocitric acids (solution 1), $\alpha-D_{1}-\alpha$-hydroxyglutaric and $\alpha-D_{1}$-malic acids made from $\alpha$-ketoglutaric and oxaloacetic acids by reduction with $\mathrm{NaBD}_{4}$ (solution 2), lactic acid (solution 3), and $\alpha-\mathrm{D}_{1^{-}}$ lactic acid prepared by reducing pyruvic acid with $\mathrm{NaBD}_{4}$ (solution 4). These four solutions were prepared in triplicate (12 stock solutions). Thirteen serial dilutions were prepared from each stock solution ranging from 0.002 to $100 \mathrm{nmol}$ per intermediate. A volume of $2 \mu \mathrm{L}$ of $3,3,3-\mathrm{D}_{3^{-}}$ lactic acid, $2 \mu \mathrm{L}$ of the internal standard mixture (Table 1 ), and $1 \mu \mathrm{L}$ of $\mathrm{D}_{27}$-myristic acid were added to each serial dilution sample. Calibration samples were then dried by vacuum centrifugation at $-4{ }^{\circ} \mathrm{C}$. Samples were re-dissolved in $30 \mu \mathrm{L}$ anhydrous pyridine, transferred to autosampler vials containing $70 \mu \mathrm{L}$ MTBSTFA and incubated at $70{ }^{\circ} \mathrm{C}$ for $1 \mathrm{~h}$ before being submitted for GC/MS data acquisition as described in the Sect. 2.6.

\section{$2.6 \mathrm{GC} / \mathrm{MS}$ analytical conditions}

GC/MS analyses were performed with an Agilent 5975C mass selective detector coupled to a 7890A gas chromatograph (Agilent Technologies, Santa Clara, CA, USA) fitted with a 7693 autosampler and a DB-5MS + DG capillary column $\left(30 \mathrm{~m}\right.$ plus $10 \mathrm{~m}$ Duraguard $\left.{ }^{\circledR}\right)$, diameter $0.25 \mathrm{~mm}$, film thickness $0.25 \mu \mathrm{m}$ (Agilent $\mathrm{J} \& \mathrm{~W}$, Santa Clara, CA, USA). The GC temperature program started with a $1 \mathrm{~min}$ hold at $120{ }^{\circ} \mathrm{C}$ followed by a $10{ }^{\circ} \mathrm{C} / \mathrm{min}$ ramp to $300{ }^{\circ} \mathrm{C}$. Bake-out was at $320^{\circ} \mathrm{C}$ for $10 \mathrm{~min}$. The injector and interface to the MS were held at $285^{\circ} \mathrm{C}$. The helium carrier flow rate was held constant at $1.5 \mathrm{~mL} / \mathrm{min}$ (or a flow rate such that the TBDMS derivative of to $\mathrm{D}_{27}$-myristic acid has a retention time of $12.4 \mathrm{~min}$ ). When operated in full scan mode, the scan range was 50-700 Da.

The GC/MS was operated in electron ionization mode $(70 \mathrm{eV})$ and in SIM (monitored ions are listed in Table 2). In most cases, the heaviest ion in each monitored group was the $\left[\mathrm{M}^{+\cdot}-\mathrm{C}_{4} \mathrm{H}_{9}\right]^{+}$fragment, which was used as the quantitating ion, while the second ion was used for verification. It should be noted that in the cases of citric and isocitric acids, the quantitating ion, $\mathrm{m} / \mathrm{z} 459$, is apparently
Table 2 Fragment ions monitored for quantitating purposes

\begin{tabular}{|c|c|c|c|}
\hline Acid & $\begin{array}{l}\text { Fragments } \\
(\mathrm{m} / \mathrm{z})\end{array}$ & $\begin{array}{l}\text { Retention } \\
\text { time (min) }\end{array}$ & $\begin{array}{l}\text { SIM interval } \\
(\min )\end{array}$ \\
\hline 3,3,3-D - -lactic & 264,236 & 6.34 & $5.50-8.00$ \\
\hline $\mathrm{D}_{1}$-lactic & 262,234 & 6.36 & $5.50-8.00$ \\
\hline Lactic & 261,233 & 6.36 & $5.50-8.00$ \\
\hline $\mathrm{D}_{4}$-succinic & 293,335 & 9.26 & $8.70-10.00$ \\
\hline Succinic & 289,331 & 9.29 & $8.70-10.00$ \\
\hline $\mathrm{D}_{2}$-fumaric & 289,331 & 9.64 & $8.70-10.00$ \\
\hline Fumaric & 287,329 & 9.65 & $8.70-10.00$ \\
\hline $\mathrm{D}_{3}$-malic & 422,290 & 12.67 & $12.00-13.00$ \\
\hline $\mathrm{D}_{1}$-malic & 420,289 & 12.68 & $12.00-13.00$ \\
\hline Malic & 419,287 & 12.68 & $12.00-13.00$ \\
\hline $\mathrm{D}_{5}-\alpha$-hydroxyglutaric & 438,249 & 13.59 & $13.00-14.00$ \\
\hline $\mathrm{D}_{1}-\alpha$-hydroxyglutaric & 434,246 & 13.61 & $13.00-14.00$ \\
\hline$\alpha$-hydroxyglutaric & 433,245 & 13.61 & $13.00-14.00$ \\
\hline Tricarballylic & 461,503 & 14.98 & $14.00-16.30$ \\
\hline cis-Aconitic & 459,501 & 15.08 & $14.00-16.30$ \\
\hline $\mathrm{D}_{4}$-citric & $462,434,406$ & 16.83 & $16.30-18.00$ \\
\hline Citric & $459,431,403$ & 16.84 & $16.30-18.00$ \\
\hline $\mathrm{D}_{1}$-isocitric ${ }^{\mathrm{a}}$ & $460,432,404$ & 16.94 & $16.30-18.00$ \\
\hline Isocitric & $459,431,403$ & 16.94 & $16.30-18.00$ \\
\hline
\end{tabular}

${ }^{a} \mathrm{D}_{1}$-isocitric is monitored (reduced oxalosuccinic). However, no authentic internal standards are available for oxalosuccinic acid, nor have we successfully measured ion intensities nominally for $\mathrm{D}_{1}$-isocitric reliably greater than those expected for the $\mathrm{M}+1$ natural abundance of isocitric acid. We include it here for completeness

the $\left[\mathrm{M}^{+\cdot}-\mathrm{C}_{4} \mathrm{H}_{9}-\right.$ tert $\left.-\mathrm{C}_{4} \mathrm{H}_{9}\left(\mathrm{CH}_{3}\right)_{2} \mathrm{SiOH}\right]$ fragment of the tetrakis-TBDMS derivative. The hydroxyl hydrogen on the TBDMS-OH comes from one of the methylene groups in the citric and isocitric backbones, and the structure of the resulting ion $(\mathrm{m} / \mathrm{z} 459)$ may resemble the $M-57$ ion for aconitic acid. However in citric $\mathrm{D}_{4}$, TBDMS-OD is lost (133 Da) and so the ion monitored for citric " $\mathrm{D}_{4}$ " contains only 3 deuteriums, and correction for natural abundance heavy isotope inclusion in unlabeled citric at $\mathrm{m} / \mathrm{z} 462$, requires the $M+3$ value. $M / z 403$ was monitored to confirm the assignment of citric and isocitric acids; the former has a much less intense m/z 403 fragment than the latter.

Monitored ion intensities were integrated and exported into a spreadsheet using MSD Chemstation software and "RTE" integrator (rev E.02.02.1431, Agilent Technologies, Santa Clara, CA, USA), enabling corrections to determine the fraction of the $\mathrm{M}+1$ ion intensities of lactic, malic, $\alpha$-hydroxyglutaric and isocitric acids due to the intensities of the deuterium-labeled ions resulting from $\mathrm{NaBD}_{4}$ reduction of pyruvic, oxaloacetic, $\alpha$-ketoglutaric and oxalosuccinic, respectively. Lactic acid requires an additional commentary, since on our GC/MS we have a constant lactic background signal. For an absolute measure 
of lactic acid in the cells this background signal should be subtracted.

\subsection{Statistical analyses}

Results from DMSO (control) and toxin treated cells were compared by one-way ANOVA with Dunnett's multiple pair-wise comparison tests using significance threshold of 0.05. Calculations were made using GraphPad Prism software (GraphPad Software, San Diego, CA, USA).

\section{Results and discussion}

\subsection{Post data acquisition calculations and corrections}

After the GC/MS data had been collected and the integrated areas for each metabolite tabulated, corrections were made to the areas as described previously (Mamer 1988; Mamer et al. 1986). Briefly, for an endogenous metabolite "A", a $\mathrm{NaBD}_{4}$ reduced mono-deuterated endogenous metabolite "A-D $\mathrm{D}_{1}$ " and isotopically labeled internal standard " $\mathrm{A}-\mathrm{D}_{\mathrm{x}}$ " (malic, $\mathrm{D}_{1}$-malic and $\mathrm{D}_{3}$-malic for example), we took into account and corrected for the natural abundance of each detected ion of the isotopic cluster and the isotopic impurities introduced by the $\mathrm{NaBD}_{4}$ reduction or the isotopically labeled internal standard. Reducing authentic unlabeled keto acids and measuring their mass spectra allowed for the determination of the isotopic impurity of the $\mathrm{NaBD}_{4}$. The isotopic impurity contribution from $A-D_{1}$ to the $A$ integrated intensities was found to be $0.6 \%$ of the $A-D_{1}$ integrated intensity. It is important to note that isotopic impurity will be specific to each lot of $\mathrm{NaBD}_{4}$ used for reduction. The stable isotope standards were also analyzed for isotopic impurity at $1 \times, 2 \times$ and $3 \times$ the amount spiked into samples. At these levels, we were not able to detect any significant unlabeled contribution in the stable isotope labeled internal standards $\left(A-D_{x}\right)$. Every new standard purchased will need to be assayed for its isotopic impurity levels. The measured peak areas for such an $I, I_{+1}$ and $I_{+x}$ cluster can be expressed as and are drawn schematically in Fig. 1:

$I=R+f_{\text {imp }} R_{+1}+f_{\text {impX } 0} R_{+x}$

$I_{+1}=R_{+1}+f_{(M+1)} R+f_{\text {impX1 }} R_{+x}$

$I_{+x}=R_{+x}+f_{(M+X)} R+f_{(D M+X)} R_{+1}$

Parameters in the equations above are defined as follows: $R$ the correct integrated area for the endogenous metabolite "A", $R_{+1}$ the corrected value for the reduced $\alpha$-keto acid "A- $\mathrm{D}_{1}$ ", $R_{+x}$ the corrected value for the isotopically labeled internal standard "A-D ${ }_{\mathrm{X}}$ ", $f_{\text {imp }}$ the isotopic impurity introduced from the $\mathrm{NaBD}_{4}$ reduction of the $\alpha$-keto acid, $f_{\text {imp } X 0}$

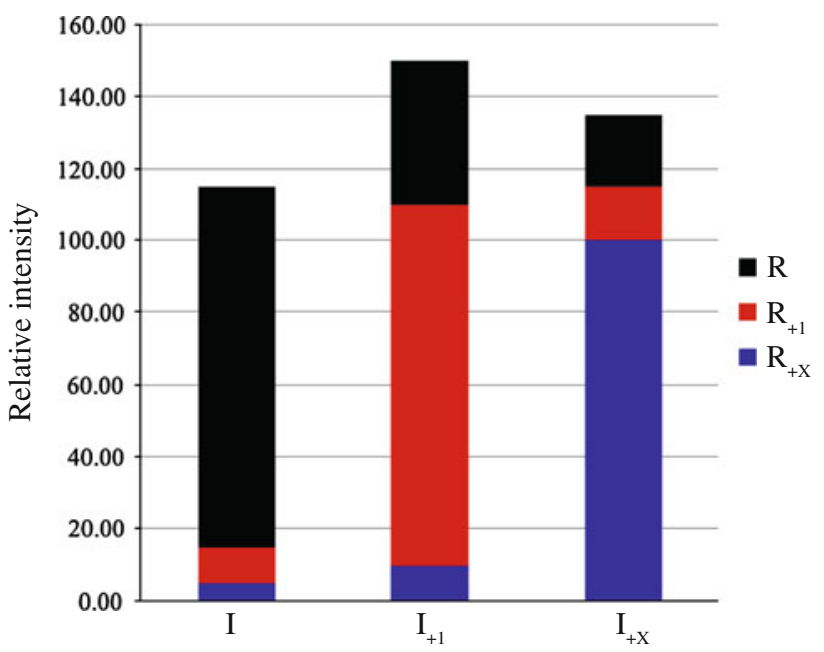

Fig. 1 A schematic diagram illustrating the natural abundance isotopic and isotopic impurity contributions expected for peak areas $I, I_{+1}$ and $I_{+x}$. The values for $R$ (black), $R_{+1}($ red $)$ and $R_{+X}($ blue $)$ were each set to 100 while the values for $f_{\text {imp }}, f_{\text {impX0 }}, f_{\text {impX1 }}, f_{(M+1)}$ and $f_{D(M+1)}$ were arbitrarily set to $10,5,10,40,20$ and $15 \%$ respectively. The multiplication factors are measured experimentally for isotopic impurities and calculated for natural isotopic abundances

the isotopic impurity introduced by the addition of the isotopically labeled standard to the $I$ peak area, $f_{\text {im } X 1}$ the isotopic impurity introduced by the labeled standard to the $I_{+1}$ peak area, $f_{(M+1)}$ the fractional value of natural isotopic abundance of the first isotopic of $\mathrm{A}, f_{(M+X)}$ the fractional value of natural isotopic abundance of the Xth isotope of $\mathrm{A}, f_{(D M+X)}$ the fractional value of the natural isotopic abundance of the Xth isotope of the $\mathrm{A}-\mathrm{D}_{1}$ reduced $\alpha$-keto acid.

The fractional isotope values can be calculated or measured experimentally using authentic samples. Thus, there are nominally three equations and three unknowns, but since the contribution of isotopic impurity from the internal standard is negligible in the present example, one can solve for $R$ and $R_{+1}$ using simple substitution (two equations with two unknowns). Isotopic ratios were measured experimentally and calculated using an online calculator (http://yanjunhua.tripod.com/pattern1.htm), or the program "Isoform ${ }^{\odot}$ " (v1.02, NIST 1997). The experimentally measured isotopic ratios were within $2 \%$ of those calculated. The corrections used for the data presented in this manuscript are as follows:

Lactic, $\mathrm{D}_{1}$-lactic, $\mathrm{D}_{3}$-lactic

$$
\begin{aligned}
& R_{261}=\left(1.0014 * I_{261}\right)-\left(0.0060 * I_{262}\right) \\
& R_{262}=\left(1.0014 * I_{262}-\left(0.2285 * I_{261}\right)\right. \\
& R_{264}=I_{264}-\left(0.0146 * R_{261}\right)-\left(0.0960 * R_{262}\right)
\end{aligned}
$$

Malic, $\mathrm{D}_{1}$-malic, $\mathrm{D}_{3}$-malic

$R_{419}=\left(1.0021 * I_{419}\right)-\left(0.0060 * I_{420}\right)$ 
$R_{420}=\left(1.0021 * I_{420}\right)-\left(0.3487 * I_{419}\right)$

$R_{422}=I_{422}-\left(0.0408 * R_{419}\right)-\left(0.1704 * R_{420}\right)$

$\alpha$-Hydroxyglutaric, $\mathrm{D}_{1}$ - $\alpha$-hydroxyglutaric, $\mathrm{D}_{5}$ - $\alpha$-hydroxyglutaric

$R_{433}=\left(1.0022 * I_{433}\right)-\left(0.0060 * I_{434}\right)$

$R_{434}=\left(1.0022 * I_{434}\right)-\left(0.3677 * I_{433}\right)$

$R_{438}=I_{438}-\left(0.0018 * R_{433}\right)-\left(0.0104 * R_{434}\right)$

Isocitric, $\mathrm{D}_{1}$-isocitric $\left(\mathrm{D}_{4}\right.$-citric is used as the internal standard as it is well resolved from isocitric)

$R_{459}=\left(1.0023 * I_{459}\right)-\left(0.0060 * I_{460}\right)$

$R_{460}=\left(1.0023 * I_{460}\right)-\left(0.3829 * I_{459}\right)$

After these corrections were made, the amount of each metabolite could be calculated from the ratio of the corrected peak areas multiplied by the stable isotopologue concentration (e.g. [Lactic] $=\mathrm{R}_{261} / \mathrm{R}_{264} *\left[\mathrm{D}_{3}\right.$-lactic]). The results from the cell extractions were expressed as nanomoles per million cells.

We selected deuterium labeled internal standards over those with ${ }^{13} \mathrm{C}$ labeling as the former are more widely available and less expensive than the latter. In our experience, the sites of deuterium labeling in the chosen internal standards are refractory to exchange at room temperature and in weakly acidic solutions (experimentally confirmed, data not shown).

\section{$3.2 \alpha$-Keto acid stability}

A small amount of each $\alpha$-ketoglutaric, oxaloacetic and pyruvic acid was directly added to $30 \mu \mathrm{L}$ of pyridine, derivatized with MTBSTFA and analyzed as described in the experimental section. Pyruvic acid gave the expected bis-TBDMS derivative along with a very small secondary peak, the nature of which we could not identify. $\alpha$-Ketoglutaric acid gave three peaks corresponding to bis-, trisand tetrakis-TBDMS derivatives. A small amount of succinic acid was also detected ( $\sim 2 \%$ by integral comparison). Interestingly, the oxaloacetic acid did not appear to be stable under these conditions. A very small peak of the tris-TBDMS derivative of oxaloacetic acid was detected in addition to a very large pyruvic acid peak. The same approach was taken again, except samples were methoximated in pyridine before derivatization and GC/MS data collected as described in the Supplementary Materials. Methoximated $\alpha$-ketoglutaric formed the expected single peak with a slightly lower level of succinic acid $(0.5 \%$ by integral comparison). The oxaloacetic methoxime also formed one peak, along with a pyruvic peak $(\sim 15 \%$ by integral comparison). For a quick test of stability, small amounts of pyruvic, oxaloacetic and $\alpha$-ketoglutaric acids were dissolved individually in $20 \mu \mathrm{L}$ of basic, acidic and neutral $80 \%$ methanol mimicking the potential range of extraction conditions. Triplicate samples were held at room temperature for $2 \mathrm{~h}$. We found that $\alpha$-ketoglutaric acid decarboxylated to a greater extent under basic compared with acidic conditions, while pyruvic acid appears to degrade to a greater extent under acidic conditions compared to neutral and basic conditions. Oxaloacetic acid appears equally unstable under all conditions tested (Supplemental Fig. S1). The decarboxylation of oxaloacetic acid to pyruvic has been noted by Kubota et al. (2005) and shown by Tsai (1967) to have a first order reaction rate constant of $7.23 \times 10^{-3} \mathrm{~min}^{-1}$ at $30{ }^{\circ} \mathrm{C}$ in water.

Furthermore, we measured how quickly $\mathrm{NaBD}_{4}$ reduction of the $\alpha$-keto acids took place compared to their decarboxylation. The reaction kinetics (described in Supplemental Materials) were assumed to be pseudo-first order in $\alpha$-keto acid due to the excess amount of $\mathrm{NaBD}_{4}$. The rate of reduction for the $\alpha$-keto acids ranged from 0.3 to $1.3 \mathrm{~min}^{-1}$ at room temperature over four independent replicate reactions (see Supplemental Fig. S2). The rate of oxaloacetic acid reduction may be faster but we were unable to collect time point aliquots faster than every 20-30 s (see Supplemental Fig. S2 B). These rates should be considered approximate since they are heavily dependent upon the amount of $\mathrm{NaBD}_{4}$ compared to the $\alpha$-keto acid amount. Under our reaction conditions, $\mathrm{NaBD}_{4}$ is added in five fold molar excess to ensure pseudo-first order rate conditions. Under the conditions of these analyses, the rate of reduction is over forty to two hundred times faster than the rate of decarboxylation reported by Tsai (1967).

In order to ensure that the extraction conditions did not cause excessive decarboxylation, we tested three different extraction conditions in triplicate, namely $80 \%$ cold methanol, basic $80 \%$ cold methanol (pH 11) and basic $80 \%$ cold methanol to which $15 \mu \mathrm{L}$ of $5 \mathrm{mg} / \mathrm{mL}$ of $\mathrm{NaBD}_{4}$ were added before the cells were lysed by sonication. The levels of each quantified CAC acid did not change significantly with different conditions, ensuring that the simple $80 \%$ methanol extraction does not cause significant decarboxylation so long as samples are kept as cold as possible up to the point of reduction (see Supplemental Fig. S3). Additionally, we do not perform any additional solvent based organic acid extractions to further isolate CAC organic acids in our sample preparation since the efficiency of the CAC organic acid extraction depends on the amount of the acid present in the crude extract (Szmigielska et al. 1995). Based on the evidence presented, we are confident that our methodology minimizes the loss of $\alpha$-keto acids to decarboxylation and the levels of quantified $\mathrm{CAC}$ organic acid intermediates accurately reflect those of the cell or tissue. 
Table 3 Upper and lower limits of quantitation for CAC organic acid intermediates

These limits of detection reflect the current state of our GC/MS system and can change over time-see text

${ }^{a}$ Uncorrected for background lactic

\begin{tabular}{lclll}
\hline CAC organic acid & $\begin{array}{l}\text { Upper limit } \\
\text { (nmol) }\end{array}$ & $\begin{array}{l}\text { Lower limit } \\
\text { (nmol) }\end{array}$ & $\begin{array}{l}R^{2} \text { curve fit } \\
\text { (linear/log-log) }\end{array}$ & $\begin{array}{l}\text { M+1/M standard deviation } \\
\text { for non-reduced } \alpha \text {-hydroxy } \\
\text { acids }\end{array}$ \\
\hline Lactic & 80 & $0.5^{\mathrm{a}}$ & $0.99 / 0.99$ & 0.006 \\
$\mathrm{D}_{1}$-lactic (pyruvic) & 25 & 0.01 & $0.96 / 0.96$ & \\
Succinic & 100 & 0.01 & $0.99 / 0.99$ & \\
Fumaric & 75 & 0.05 & $0.99 / 0.99$ & \\
Malic & 75 & 0.05 & $0.99 / 0.99$ & 0.004 \\
$\mathrm{D}_{1}$-malic (oxaloacetic) & 60 & 0.01 & $0.99 / 0.99$ & \\
$\alpha$-Hydroxyglutaric & 100 & 0.01 & $0.99 / 0.99$ & 0.005 \\
$\mathrm{D}_{1}$ - $\boldsymbol{\alpha}$-hydroxyglutaric & 75 & 0.01 & $0.98 / 0.96$ & \\
$\quad(\alpha-$ ketoglutaric) & & & & \\
cis-Aconitic & 75 & 0.01 & $0.99 / 0.99$ & 0.002 \\
Citric & 100 & 0.01 & $0.99 / 0.99$ & \\
Isocitric & 100 & 0.01 & $0.99 / 0.99$ & \\
\hline
\end{tabular}

\subsection{Limits of quantitation}

The limits of quantitation (LOQ) were measured for all of the CAC organic acids. A summary of these limits is found in Table 3. The calibration curves measured in triplicate from freshly prepared solutions can be found in Supplementary Fig. S4. The upper and lower limits of quantitation are dynamic and depend on the physical status of the mass spectrometer. Some factors that can contribute to loss of sensitivity and dynamic range are the cleanliness of the ion source, the quadrupoles, lenses and age of the detector. Because this methodology uses stable isotope dilution techniques, the isotope corrected integral values for the internal standards should remain relatively constant from sample to sample.

In the case of the upper limit, if the corrected integral of the stable isotope internal standard associated with the organic acid of interest is much larger than that of the other samples (or samples containing only the internal standards), then the upper limit for that particular metabolite has been exceeded and the sample should be diluted and re-run. In this case the metabolite peak is truncated since the electron multiplier output is no longer proportional to ion current input thus compromising the isotopic ratios.

For the lower limit of non-reduced metabolites, the chromatogram must be examined to determine if the lowlevel peak signal is sufficiently above the noise level $(\mathrm{S} / \mathrm{N}$ 10:1). Under low resolution GC/MS conditions, the $\alpha$ hydroxy acids produced by the reduction of the four $\alpha$-keto acids with sodium borodeuteride will co-elute with the corresponding four endogenous unlabeled $\alpha$-hydroxy acids, and their $[\mathrm{M}-57]^{+}$fragments will have the same nominal masses as the $[\mathrm{M}-56]^{+}$first isotope peaks of the unlabeled $\alpha$-hydroxy acids. In all four cases, the former may be less intense than the latter, and the limits of quantitation for the $\alpha$-keto acids will then be related to the standard deviations measured for the intensities of the $[\mathrm{M}-56]^{+}$ions in technical replicates of the unlabeled $\alpha$-hydroxy acids (Table 3 ). We assume that the lower limit of quantitation is reached when the analyte signal is less than 10 times the noise, where the 'noise' is defined as the standard deviation for the intensity of the $[M-56]^{+}$in each of the unlabeled $\alpha-$ hydroxy acids. The standard deviations measured are listed in Table 3. We report the LOQ for the CAC acids as the points where the calibrating plots depart significantly from linearity at the high and low ends.

\subsection{Mitochondrial toxin perturbation of the citric acid cycle}

Figure 2 shows the selected ion chromatograms for the authentic standards and extracts of cells exposed to DMSO (control), antimycin A, rotenone, and oligomycin. It is important to appreciate that all targeted metabolites are well resolved including citric and isocitric acids. The reduced mono-deuterated species and internal standards co-elute with their corresponding non-deuterated metabolites (Fig. 2).

Figure 3 shows box and scatter plots of CAC intermediates measured for five replicates. All of the CAC organic acid intermediates were successfully quantified except for oxalosuccinic acid (which would be reduced to $\mathrm{D}_{1}$-isocitric acid). We have not detected oxalosuccinic acid as $\mathrm{D}_{1}$-isocitric acid in various cell lines and tissue extracts (data not shown). It is likely to be below the limits of detection, unstable during the cell extraction or precipitated along with the enzyme, isocitric dehydrogenase, to which it is reported to be tightly bound (Siebert et al. 1957). Oxalosuccinic acid has, however, been measured in hydrogenobacter thermophylus TK-6 (Aoshima and Igarashi 2006). 

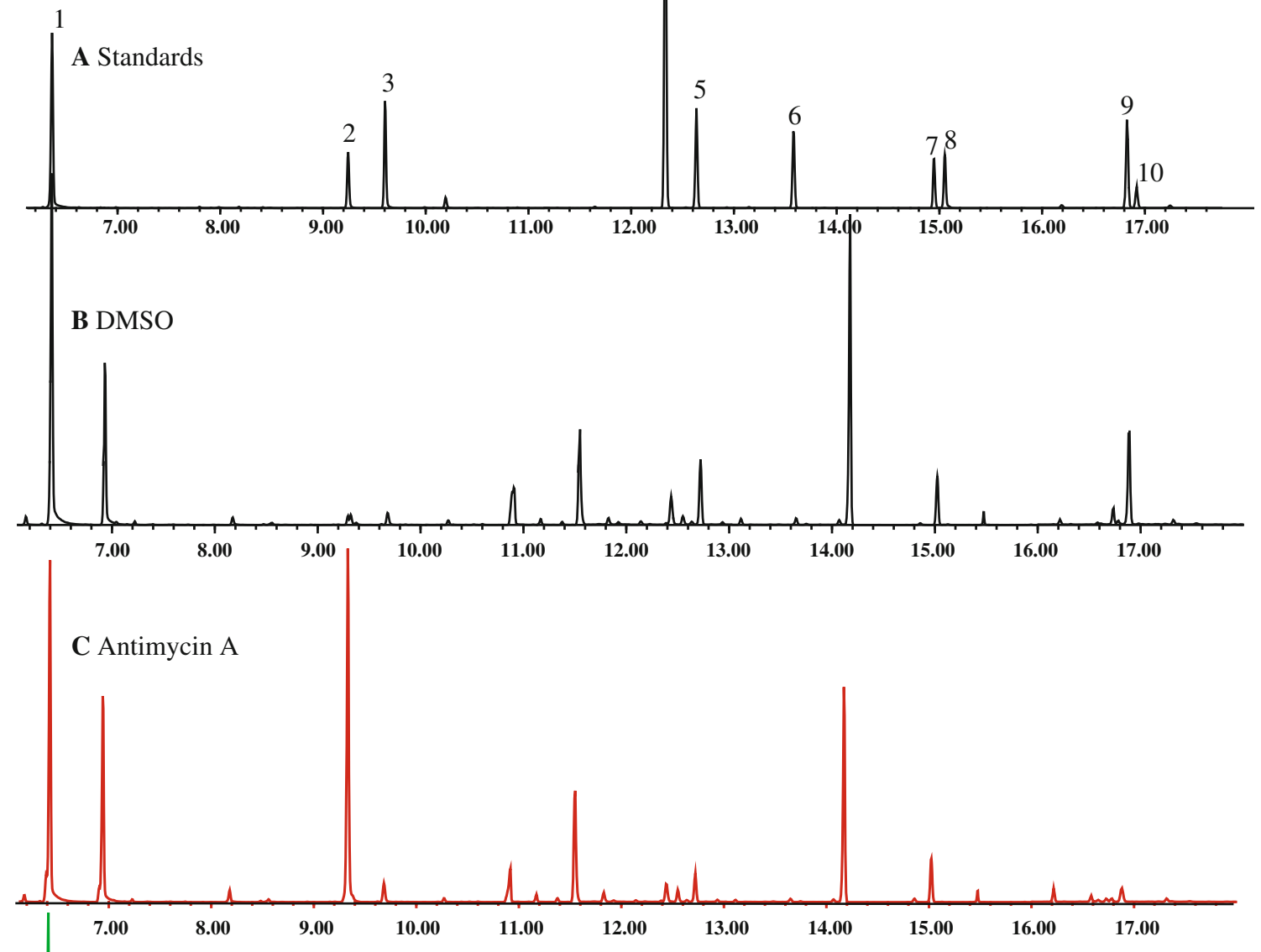

D Rotenone
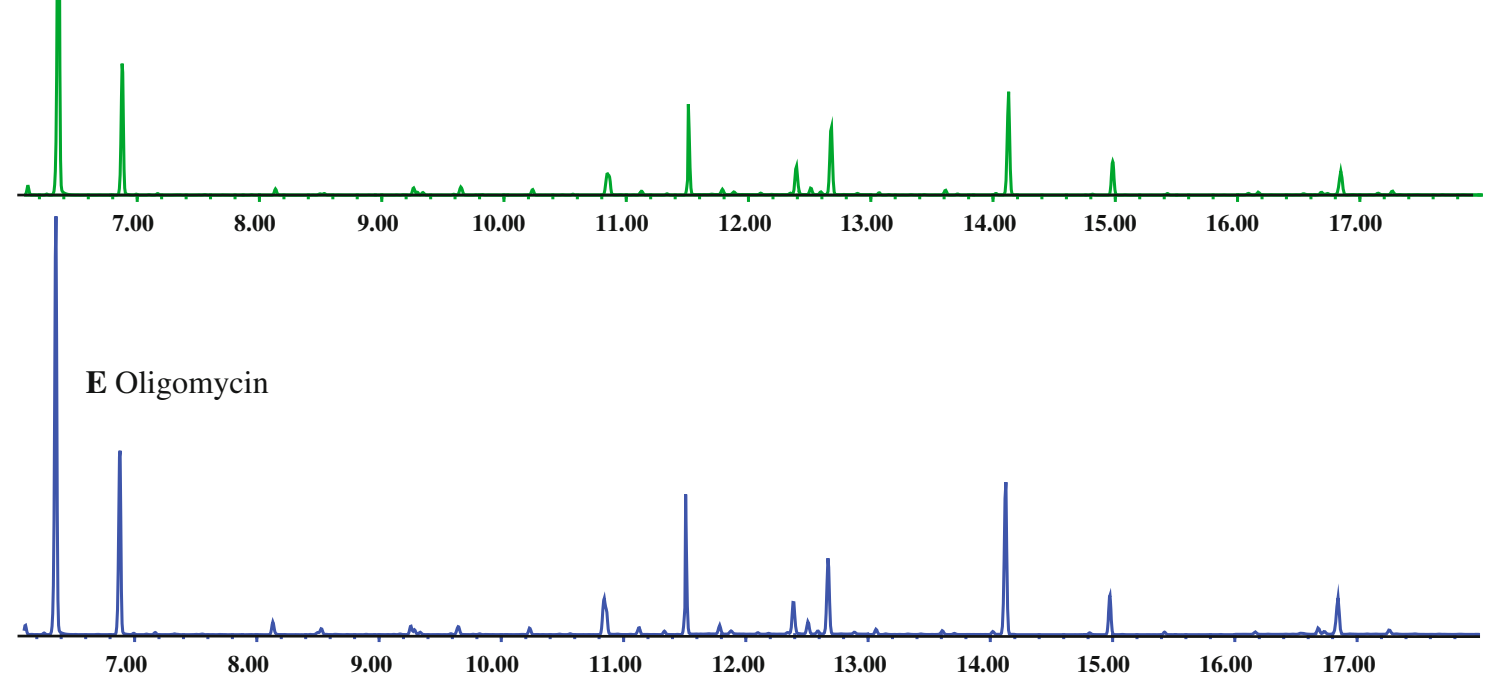
4Fig. 2 The selected ion current chromatograms for a authentic standards, b DMSO treated cells, $\mathbf{c}$ antimycin A treated cells, $\mathbf{d}$ rotenone treated cells, and e oligomycin treated cells. Selected ion monitoring data were collected for analytical samples using ions listed in Table 2. Numbers above authentic standard peaks represent unlabeled and reduced deuterium-labeled 1 lactic and $\mathrm{D}_{1}$-lactic, 2 succinic, 3 fumaric, $4 \mathrm{D}_{27}$-myristic, 5 malic and $\mathrm{D}_{1}$-malic, $6 \alpha$-hydroxyglutaric and $\alpha-\mathrm{D}_{1}$ hydroxyglutaric, 7 tricarballylic, $8 \mathrm{cis}$-aconitic, 9 citric and 10 isocitric and $\mathrm{D}_{1}$-isocitric acids

As a first proof of principle, we wanted to quantify the lactic acid concentration of control cells and cells exposed to mitochondrial toxins. These mitochondrial drugs are expected to halt ATP production, and the cells compensate by increasing flux through the glycolytic pathway, pyruvate to lactate, as observed in Fig. 3. A schematic of the CAC along with bar graphs of mitotoxin response normalized to DMSO control metabolite levels are shown in Fig. 4 for three independent cell passages.

In order to perturb the concentration of CAC intermediates, we first treated cells with antimycin $\mathrm{A}$, an inhibitor of complex III. It is expected that a blockade of the electron transport chain at complex III will result in an accumulation of upstream metabolites, notably $\mathrm{FADH}_{2}$, which in
Fig. 3 Typical analysis of citric acid cycle organic acid intermediates quantified by selected ion monitoring GC/MS for a single cell passage and five replicates for each treatment.

The distributions are displayed as box plots of DMSO (black), antimycin A (red), rotenone (green), and oligomycin (blue) exposed cells normalized per million cells. Note that the plot for succinic acid contains a break in the vertical axis due to its large range in concentration. Treated groups were compared to DMSO using one-way ANOVA with Dunnett's multiple comparisons test;

$* P$ value $<0.05$

$* * P$ value $<0.01$

$* * * P$ value $<0.001$
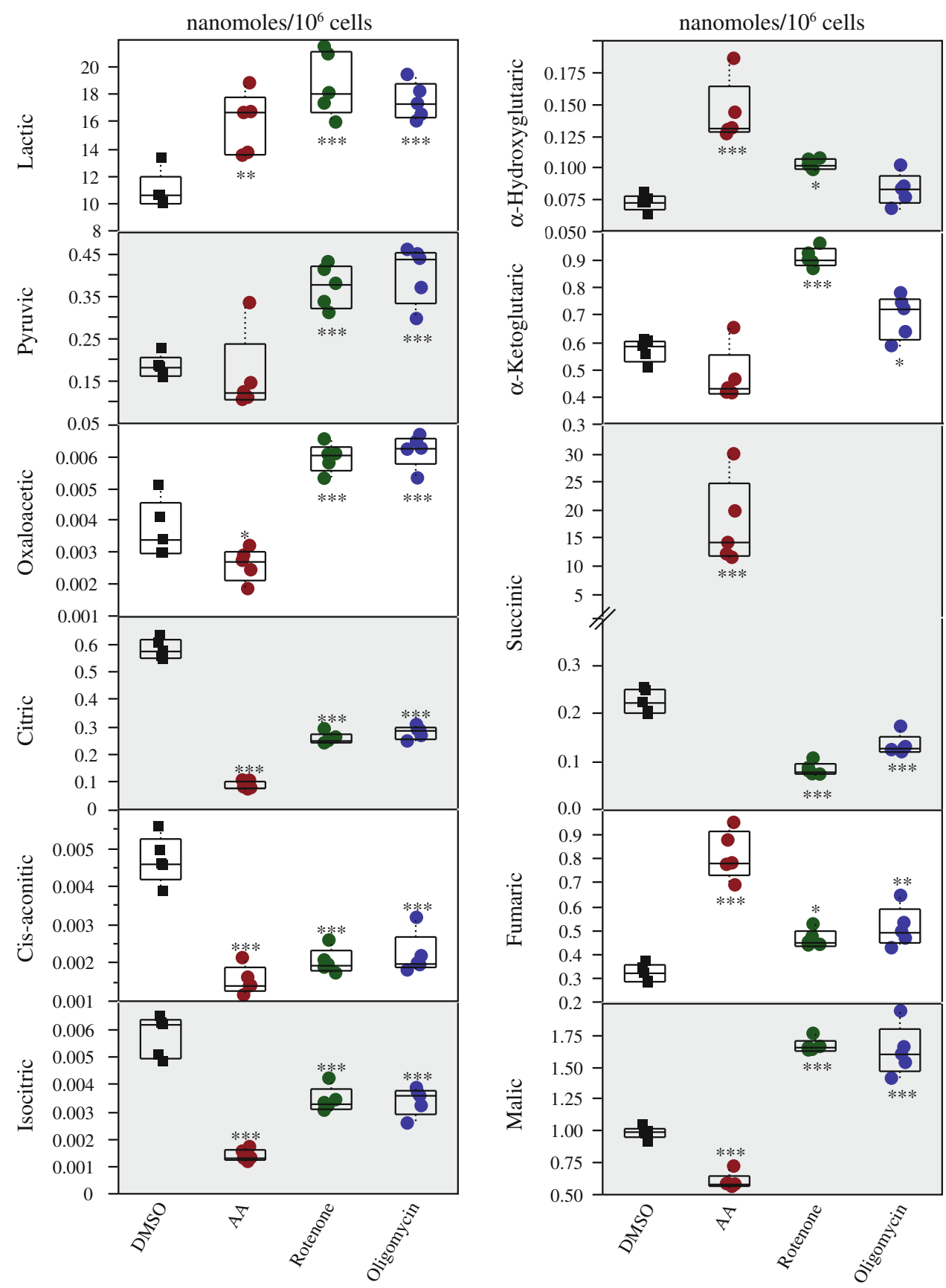


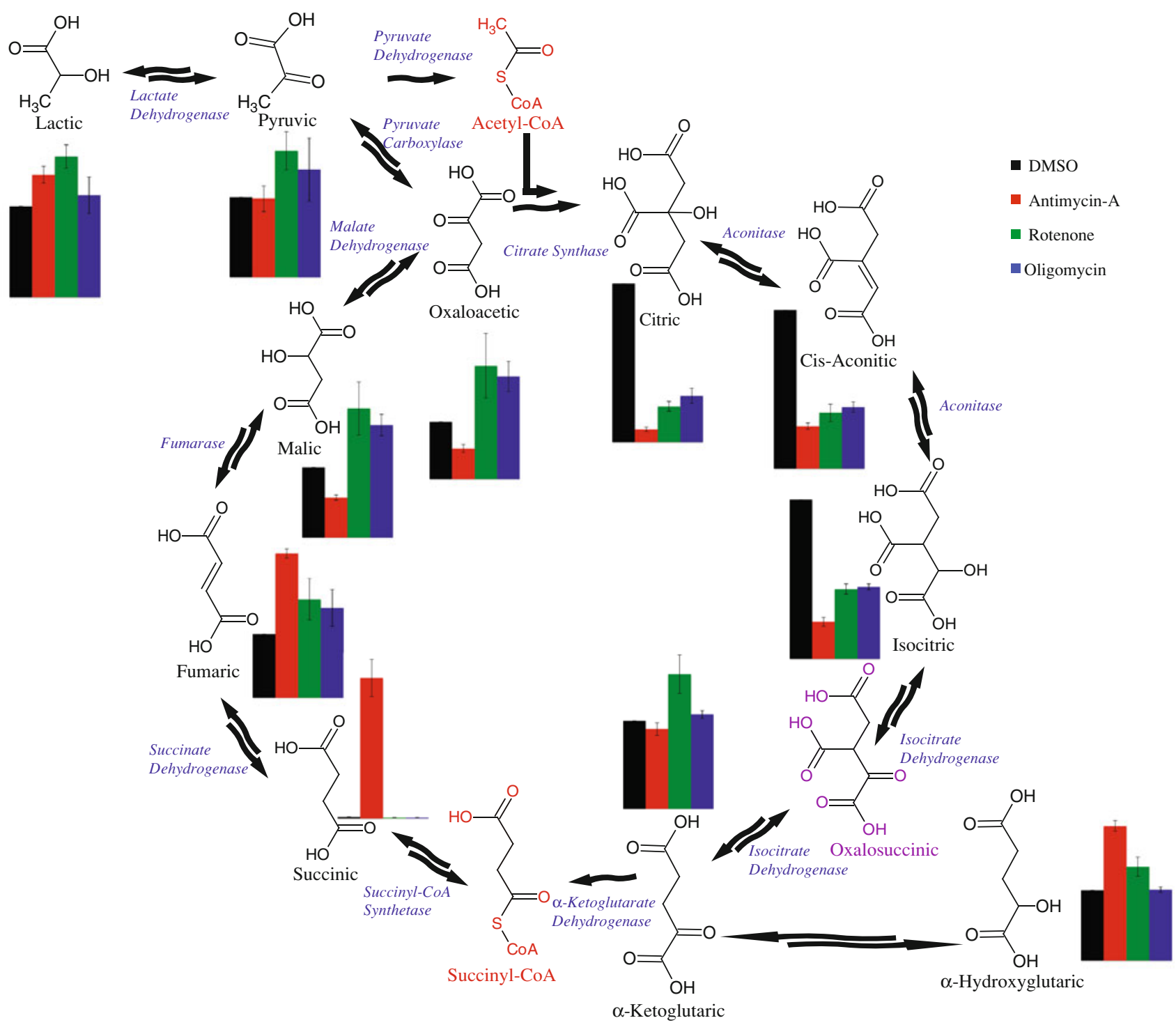

Fig. 4 A schematic of the citric acid cycle. The small bar graphs associated with each organic acid represent the level of each metabolite detected normalized against the control DMSO treatment. The error bars represent the standard error of the mean (SEM) for three separate cell passages. The cell treatments are indicated as black DMSO, red antimycin A, green rotenone, and blue oligomycin.

turn will lead to an accumulation of succinic acid by reversal of the succinate dehydrogenase reaction. Indeed, cells treated with antimycin A displayed large increases in succinic compared with controls (Figs. 3, 4). Treatment of cells with antimycin A is also expected to result in the accumulation of NADH, which is upstream of complex III in the electron transport chain. Elevated NADH levels will cause the inhibition of three CAC enzymes, citrate synthase, isocitrate dehydrogenase and $\alpha$-ketoglutarate dehydrogenase, in addition to halting the pyruvate dehydrogenase complex, which provides acetyl coenzyme A for the citrate synthase reaction. In agreement with this,
A quantitative visualization of the measured data is displayed in Fig. 3. Acetyl- and succinyl-CoAs (indicated in red) are not measured by the technique described here. As discussed in the text, oxalosuccinic acid (indicated in purple), which is reduced by sodium borodeuteride, was not detected as $\mathrm{D}_{1}$-isocitric acid, but is included here for completeness

cells treated with antimycin A had reduced concentrations of citric, cis-aconitic and $\alpha$-ketoglutaric acids compared to control cells (Figs. 3, 4). A complementary explanation for these results is that an increased redox state can lead to the reversal of the citric acid cycle, whereby amino acids are converted to $\alpha$-ketoglutaric acid, which in turn, is converted to isocitric acid and from this point the cycle is running in a counter-clockwise direction (Metallo et al. 2012; Mullen et al. 2012). In support of this point, cells treated with antimycin A exhibited an accumulation of $\alpha$-hydroxyglutaric acid, a metabolite that can be generated through the non-carboxylating reduction of $\alpha$-ketoglutaric 
(Figs. 3, 4) (Wise et al. 2011). The reduced levels of CAC intermediates upstream of $\alpha$-ketoglutaric acid during reverse activity would be explained by the fact that citric acid is diverted from the cycle for lipid biosynthesis (Filipp et al. 2012; Metallo et al. 2012; Mullen et al. 2012). Finally, it is important to point out that during the diversion of CAC intermediates for biosynthesis reactions, pyruvate carboxylase can help replenish those intermediates by converting pyruvic to oxaloacetic acid. In agreement with this point, antimycin A led to a similar change in the profiles of pyruvic and oxaloacetic acids (Figs. 3, 4).

The second treatment to perturb the CAC intermediates was to treat cells with rotenone, a complex I inhibitor that will cause accumulation of NADH. As mentioned before, NADH globally limits CAC activity by inhibiting three of its enzymes as well as the pyruvate dehydrogenase complex, which provides the starting material for the cycle, acetyl coenzyme A. In support of the blockade of the initial steps of the citric acid cycle, cells treated with rotenone displayed reduced concentrations of citric, cis-aconitic and isocitric acids (Figs. 3, 4). The levels of $\alpha$-ketoglutaric and $\alpha$-hydroxyglutaric acids were elevated in cells treated with rotenone compared with controls, indicating reverse CAC activity in the presence of the mitochondrial toxin (Figs. 3, 4). Finally, the levels of malic and oxaloacetic acids were higher in cells treated with rotenone than controls, likely due to a combination of the reversal of the malate dehydrogenase reaction through NADH accumulation as well as pyruvate carboxylase activity.

Lastly, we determined the impact of the ATP synthase inhibitor, oligomycin, on the concentration of the intermediates of the citric acid cycle. Oligomycin is expected to slow down the electron transport chain and the citric acid cycle. Even though oligomycin is expected to affect the electron transport chain globally, we observed that the CAC metabolite profile in the presence of this mitotoxin was most similar to that of rotenone (Figs. 3, 4), perhaps highlighting the sensitivity of the CAC to perturbation in NADH levels.

In summary, cells treated with the 3 mitochondrial drugs displayed an elevated glycolytic flux as illustrated by a significant rise in intracellular lactic acid concentration compared with DMSO controls (Fig. 3). Xu et al. (2011) report similar results for changes in lactic, malic, fumaric, and succinic acid levels in myotube cells treated with rotenone or antimycin A, but no significant changes in pyruvic, oxaloacetic or citric acids.

\section{Conclusions}

We describe a simple quantitative method for profiling all of the organic acids of the citric acid cycle, even for those that are unstable or lacking commercially available stable isotope analogues. Crude cell extracts were reduced with sodium borodeuteride, stable isotopically labeled analogues were added, and resulting samples were derivatized with MTBSTFA followed by SIM GC/MS. The resulting integrated peak areas were then corrected and compared to the peak areas of internal isotopically labeled standards. Through the use of three mitochondrial toxins, we demonstrated that this technique can be used to reveal specific signatures of CAC intermediates during inhibition of the electron transport chain complexes (Fig. 4).

Many laboratories are interested in defining mitochondrial functions in physiological and pathological conditions in a more quantitative manner. Given that the CAC is amphibolic in nature such that it plays a central role in catabolic and anabolic functions, the quantitative profiling of these intermediates is a central tool in basic or applied metabolic research.

Acknowledgments Metabolite measurements were performed at the Rosalind and Morris Goodman Cancer Research Centre Metabolomics Core Facility, supported by Génome Québec, the Canada Foundation of Innovation, The Dr. John R. and Clara M. Fraser Memorial Trust, the Terry Fox Foundation (TFF Oncometabolism Team Grant 116128 to JSP and Metabolomics Core Facility), and McGill University. SPG is supported by a postdoctoral fellowship from the Canderel Foundation. JSP is a FRSQ research scholar. We gratefully acknowledge the technical advice and expertise from Stephan Baumann and Christian Foucault (Agilent). We thank Dr. Gaëlle Bridon for the critical reading of this manuscript.

\section{References}

Aoshima, M., \& Igarashi, Y. (2006). A novel oxalosuccinate-forming enzyme involved in the reductive carboxylation of 2-oxoglutarate in Hydrogenobacter thermophilus TK-6. Molecular Microbiology, 62, 748-759. doi:10.1111/j.1365-2958.2006.05399.x.

Busch, H., Hurlbert, R. B., \& Potter, V. R. (1952). Anion exchange chromatography of acids of the citric acid cycle. The Journal of Biological Chemistry, 196, 717-727.

Chatham, J. C., Bouchard, B., \& Des Rosiers, C. (2003). A comparison between NMR and GCMS 13C-isotopomer analysis in cardiac metabolism. Molecular and Cellular Biochemistry, $249,105-112$.

Dalgliesh, C. E., Horning, E. C., Horning, M. G., Knox, K. L., \& Yarger, K. (1966). A gas-liquid-chromatographic procedure for separating a wide range of metabolites occurring in urine or tissue extracts. The Biochemical Journal, 101, 792-810.

Dunn, W. B., \& Winder, C. L. (2011). Sample preparation related to the intracellular metabolome of yeast methods for quenching, extraction, and metabolite quantitation. Methods in Enzymology, 500, 277-297. doi:10.1016/B978-0-12-385118-5.00015-3.

Fiehn, O. (2001). Combining genomics, metabolome analysis, and biochemical modelling to understand metabolic networks. Comparative and Functional Genomics, 2, 155-168. doi:10.1002/cfg.82.

Filipp, F. V., Scott, D. A., Ronai, Z. A., Osterman, A. L., \& Smith, J. W. (2012). Reverse TCA cycle flux through isocitrate dehydrogenases 1 and 2 is required for lipogenesis in hypoxic melanoma 
cells. Pigment Cell \& Melanoma Research, 25, 375-383. doi: 10.1111/j.1755-148X.2012.00989.x.

Harmon, M. A., \& Doelle, H. W. (1969). Gas chromatographic separation and determination of microquantities of the esters of the tricarboxylic acid cycle acids and related compounds. Journal of Chromatography A, 42, 157-169. doi:10.1016/s00219673(01)80611-7.

Kombu, R. S., Brunengraber, H., \& Puchowicz, M. A. (2011). Analysis of the citric acid cycle intermediates using gas chromatography-mass spectrometry. Methods in Molecular Biology, 708, 147-157. doi:10.1007/978-1-61737-985-7_8.

Koubaa, M., Cocuron, J. C., Thomasset, B., \& Alonso, A. P. (2013). Highlighting the tricarboxylic acid cycle: liquid and gas chromatography-mass spectrometry analyses of (13)C-labeled organic acids. Analytical Biochemistry,. doi:10.1016/j.ab. 2013.01.027.

Kubota, K., et al. (2005). Development of an HPLC-fluorescence determination method for carboxylic acids related to the tricarboxylic acid cycle as a metabolome tool. Biomedical Chromatography: BMC, 19, 788-795. doi:10.1002/bmc.518.

Luo, B., Groenke, K., Takors, R., Wandrey, C., \& Oldiges, M. (2007). Simultaneous determination of multiple intracellular metabolites in glycolysis, pentose phosphate pathway and tricarboxylic acid cycle by liquid chromatography-mass spectrometry. Journal of Chromatography A, 1147, 153-164. doi:10.1016/j.chroma.2007. 02.034 .

Mamer, O. A. (1988). Measurement of urinary lactic, 3-hydroxybutyric, pyruvic and acetoacetic acids in a single analysis using selected ion monitoring and stable isotope labelling techniques. Biomedical and Environmental Mass Spectrometry, 15, 57-62. doi:10.1002/bms.1200150108.

Mamer, O. A., Laschic, N. S., \& Scriver, C. R. (1986). Stable isotope dilution assay for branched chain alpha-hydroxy-and alphaketoacids: serum concentrations for normal children. Biomedical and Environmental Mass Spectrometry, 13, 553-558.

Metallo, C. M., et al. (2012). Reductive glutamine metabolism by IDH1 mediates lipogenesis under hypoxia. Nature, 481, 380-384. doi: 10.1038 /nature 10602.

Mullen, A. R., et al. (2012). Reductive carboxylation supports growth in tumour cells with defective mitochondria. Nature, 481, 385-388. doi:10.1038/nature10642.
Siebert, G., Carsiotis, M., \& Plaut, G. W. (1957). The enzymatic properties of isocitric dehydrogenase. The Journal of Biological Chemistry, 226, 977-991.

Soga, T., Ohashi, Y., Ueno, Y., Naraoka, H., Tomita, M., \& Nishioka, T. (2003). Quantitative metabolome analysis using capillary electrophoresis mass spectrometry. Journal of Proteome Research, 2, 488-494. doi:10.1021/pr034020m.

Steuer, R., Kurths, J., Fiehn, O., \& Weckwerth, W. (2003). Interpreting correlations in metabolomic networks. Biochemical Society Transactions, 31, 1476-1478. doi:10.1042/.

Szmigielska, A. M., Van Rees, K. C. J., Cieslinski, G., Huang, P. M., \& Knott, D. R. (1995). Determination of low molecular weight dicarboxylic acids in root exudates by gas chromatography. Journal of Agricultural and Food Chemistry, 43, 956-959. doi: 10.1021/jf00052a021.

Tsai, C. S. (1967). Spontaneous decarboxylation of oxaloacetic acid. Canadian Journal of Chemistry, 45, 873-880. doi:10.1139/v67-145.

Turkelson, V. T., \& Richards, M. (1978). Separation of the citric acid cycle acids by liquid chromatography. Analytical Chemistry, 50, 1420-1423. doi:10.1021/ac50033a012.

Vander Heiden, M. G., Cantley, L. C., \& Thompson, C. B. (2009). Understanding the Warburg effect: The metabolic requirements of cell proliferation. Science, 324, 1029-1033. doi:10.1126/sci ence. 1160809 .

Wakayama, M., Aoki, N., Sasaki, H., \& Ohsugi, R. (2010). Simultaneous analysis of amino acids and carboxylic acids by capillary electrophoresis, mass spectrometry using an acidic electrolyte and uncoated fused-silica capillary. Analytical Chemistry, 82, 9967-9976. doi:10.1021/ac1019039.

Wise, D. R., et al. (2011). Hypoxia promotes isocitrate dehydrogenase-dependent carboxylation of alpha-ketoglutarate to citrate to support cell growth and viability. Proceedings of the National Academy of Sciences of the United States of America, 108, 19611-19616. doi:10.1073/pnas.1117773108.

Xu, Q., Vu, H., Liu, L., Wang, T. C., \& Schaefer, W. H. (2011). Metabolic profiles show specific mitochondrial toxicities in vitro in myotube cells. Journal of Biomolecular NMR, 49, 207-219. doi:10.1007/s10858-011-9482-8.

Zaura, D. S., \& Metcoff, J. (1969). Quantification of seven tricarboxylic acid cycle and related acids in human urine by gas-liquid chromatography. Analytical Chemistry, 41, 1781-1787. 